\title{
EFEITOS DO CARBOFURANO, METSULFUROM-METÍLICO E AZINSULFUROM NA SOBREVIVÊNCIA DE CARPAS E PRODUÇÃO DE ARROZ E PEIXES EM RIZIPISCICULTURA
}

\author{
JAQUELINE INEU GOLOMBIESKI* \\ ENIO MARCHESAN** \\ EDINALVO RABAIOLI CAMARGO*** \\ GEOVANE BOSCHMANN REIMCHE**** \\ RENATO ZANELLA $A^{\star \star \star \star \star *}$ \\ LINDOLFO STORCK ${ }^{\star \star \star \star \star \star *}$
}

\begin{abstract}
Este trabalho foi conduzido com o objetivo de avaliar a sobrevivência de alevinos de carpas expostas ao carbofurano, metsulfurom-metílico e azinsulfurom em rizipiscicultura, bem como a produção de arroz irrigado e peixes. Foram utilizados alevinos de carpas húngara, capim e cabeça grande, expostos aos agroquímicos em sistema pré-germinado de produção de arroz com manejo adequado de lâmina de água contínua, durante os anos agrícolas de 2003/04 e 2004/05. Adotou-se delineamento experimental de blocos ao acaso com oito tratamentos e quatro repetições, associando carpas sob a presença ou ausência dos agroquímicos. Os alevinos de carpas foram colocados na área sete dias após a aplicação dos produtos, sendo verificado que os agroquímicos não afetaram sua sobrevivência. Devido a utilização de lâmina contínua de água no sistema, a produção de grãos de arroz também não foi afetada pela utilização de agroquímicos.
\end{abstract}

PALAVRAS-CHAVE: Cyprinus carpio; Ctenopharyngodon idella; Aristichthys nobilis; RIZIPISCICULTURA; CARBOFURANO; METSULFUROM-METÍLICO; AZINSULFUROM.

\footnotetext{
Doutora em Agronomia, Departamento de Fitotecnia, Universidade Federal de Santa Maria (UFSM), Santa Maria, RS, Brasil (e-mail golombieski@smail.ufsm.br).

** Doutor em Fitotecnia, Professor Titular, Departamento de Fitotecnia, UFSM, Santa Maria, RS (e-mail: emarch@ccr.ufsm.br).

*** Mestre, Departamento de Fitotecnia, UFSM, Santa Maria, RS (e-mail: nalvo@mail.ufsm.br).

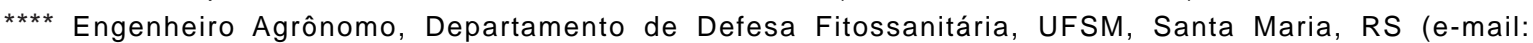
geovane_reimche@yahoo.com.br).

***** Doutor em Química Analítica, Professor Associado, Departamento de Química, UFSM, Santa Maria, RS (e-mail: rzanella@smail.ufsm.br).

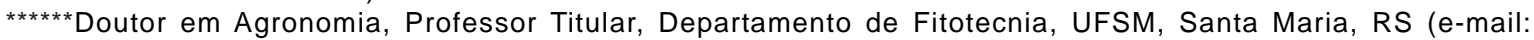
lindolfo@smail.ufsm.br).
} 


\section{INTRODUÇÃO}

O sistema pré-germinado de cultivo do arroz tem sido utilizado, principalmente em pequenas propriedades. A rizipiscicultura, alternativa para se obter produção intensiva e sustentável da área, caracteriza-se pelo cultivo consorciado de arroz irrigado e a criação de peixes, reduzindo o uso de máquinas na lavoura (COTRIM et al., 2002). As espécies de peixes mais utilizadas na rizipiscicultura são as carpas húngara (Cyprinus carpio), capim (Ctenopharyngodon idella), cabeça grande (Aristichthys nobilis) e prateada (Hypophthalmicthys molitrix). Essas espécies apresentam diferentes hábitos alimentares, sendo a carpa cabeça grande zooplanctófaga, a prateada fitoplanctófaga e a capim herbívora. A carpa húngara, de hábito alimentar omnívoro, ingere sementes, minhocas, insetos, pequenos moluscos e outros. As carpas favorecem o aproveitamento dos recursos aquáticos, diminuindo a população de plantas daninhas na cultura do arroz por se alimentarem de sementes e plantas. Além disso, revolvem o solo após a colheita do arroz e promovem correções no microrelevo.

O manejo da irrigação, mediante manutenção contínua da lâmina de água, é decisivo para o controle de gramíneas, espécies que representam o maior custo para o controle químico. Entretanto, certas espécies não são controladas por meio da lâmina de água. Esse é o caso de espécies de folhas largas e, em certas situações, espécies de ciperáceas, sendo necessária à aplicação de herbicidas. $O$ herbicida azinsulfurom é utilizado no controle de Sagittaria sp. (chapéu-de-couro), Cyperus sp. (junquinho) e Fimbristylis sp. (cuminho) e o metsulfurom-metílico para o controle de Aeschynomene sp. (angiquinho) e Heteranthera sp. (agrião-do-brejo) (SOSBAI, 2005). Para o controle de insetos como, por exemplo, larvas do gorgulho aquático Oryzophagus oryzae (bicheira-da-raiz) pode ser aplicado o inseticida carbofurano. Essa constitui a principal praga na condição de cultivo pré-germinado, podendo reduzir a produtividade da cultura do arroz em até 10\% (MARTINS et al., 2004).

É importante verificar se os agroquímicos aplicados na lavoura arrozeira afetam a população de peixes e se a produtividade da cultura do arroz é influenciada pela presença de peixes na área. Assim, o presente estudo teve como objetivo avaliar a sobrevivência de alevinos de carpas húngara, capim e cabeça grande, expostas ao carbofurano, metsulfurom-metílico e azinsulfurom em rizipiscicultura, bem como a produção de arroz irrigado e peixes na área.

\section{MATERIAL E MÉTODOS}

O experimento foi conduzido em área de várzea sistematizada da Universidade Federal de Santa Maria (UFSM), durante os anos agrícolas de 2003/04 e 2004/05, em solo classificado como Planossolo Hidromórfico eutrófico arênico. Adotou-se o delineamento experimental de blocos ao acaso (quatro repetições) e os tratamentos: $[T 1]$ azinsulfurom $(A)$ em rizipiscicultura $(P=$ peixes); [T2] metsulfurom-metílico $(M)+(P)$; [T3] carbofurano $(C)+P$; [T4] A+M+C+P; [T5] A+M+C em área somente com arroz; [T6] A+M+P; [T7] testemunha 1 Arroz (Ar) e [T8] testemunha $2(\mathrm{Ar}+\mathrm{P})$.

O primeiro ano experimental (ano 1) abrangeu de novembro/03 à outubro/04 e o segundo (ano 2) de novembro/04 à julho/05. Após o preparo da área no ano 1 foram construídas as taipas e refúgios (vala lateral para abrigar os peixes $-5,3 \mathrm{~m}$ comprimento $\times 0,7 \mathrm{~m}$ largura $\times 0,7 \mathrm{~m}$ profundidade), representando aproximadamente $8 \%$ da área total das unidades experimentais com $48 \mathrm{~m}^{-2}(8 \mathrm{~m} \times 6 \mathrm{~m})$. Antes da semeadura no ano 2 executou-se a aplicação de herbicida de ação total, visando a limpeza da área experimental. Posteriormente, procedeu-se a semeadura do arroz sem o revolvimento do solo. Nos dois anos experimentais foi realizada a calagem nas parcelas, a fim de se obter níveis adequados de dureza total e pH. A condução da água para as parcelas ocorreu mediante canal de superfície, abastecendo cada parcela individualmente. Aárea foi cercada com telas de malha $4,9 \mathrm{~cm} \times 4,9 \mathrm{~cm}$ e $0,5 \mathrm{~m}$ de altura para minimizar a entrada de animais predadores.

Semeou-se o arroz no sistema pré-germinado, utilizando $120 \mathrm{~kg} \mathrm{ha}^{-1}$ de sementes da cultivar IRGA 419 no ano 1 (18/10/03) e IRGA 420 no ano 2 (22/10/04). A área foi inundada cerca de 20 dias antes da semeadura, mantendo-se a lâmina com cerca de $0,20 \mathrm{~m}$ de altura durante todo o ciclo da cultura. Os agroquímicos foram aplicados aos 28 dias após a semeadura do arroz (DAS) nos dois anos, utilizandose azinsulfurom (5 g.i.a.ha-1 ou 0,005 $\mathrm{mg} \mathrm{L}^{-1}$ ) e metsulfurom-metílico (2 g.i.a.ha ${ }^{-1}$ ou 0,002 $\mathrm{mg} \mathrm{L}^{-1}$ ) 
aplicados pelo método de benzedura, e carbofurano (750 g.i.a.ha-1 ou 0,75 $\left.\mathrm{mg} \mathrm{L}^{-1}\right)$ distribuído à lanço. Efetuou-se a adubação de acordo com as recomendações técnicas da cultura (SOSBAl, 2003).

As proporções de peixes utilizadas foram: $60 \%$ de carpa húngara, $20 \%$ de carpa capim e $20 \%$ de carpa cabeça grande na densidade de povoamento de 20.000 alevinos ha-1 ${ }^{-1} \mathrm{Na}$ área de cada refúgio com rizipiscicultura colocou-se pequeno tanque-rede $\left(0,30 \mathrm{~m} \times 1,05 \mathrm{~m}=0,073 \mathrm{~m}^{3}\right.$ de capacidade) no dia da aplicação dos agroquímicos, sendo povoado com alevinos ( $n=6$ para cada espécie) e observada a mortalidade dos peixes pelo período de $96 \mathrm{~h}$. Os alevinos foram colocados na área experimental aos sete dias após a aplicação dos tratamentos (Tabela 1). Diariamente foi observada a presença de predadores, bem como a mortalidade de peixes na área experimental. Realizou-se experimento complementar, em laboratório, com os três agroquímicos em questão a fim de se obter a concentração letal mediana $\left(\mathrm{CL}_{50} ; 9 \mathrm{~h}\right)$ para as carpas. Assim, os índices de segurança (risco de impacto ambiental) para as três espécies foram calculados dividindo-se o valor da $\mathrm{CL}_{50 ;} 96 \mathrm{~h}$ pela concentração teórica do agroquímico na água da lavoura (SOLOMON, 1997).

\section{TABELA 1 - BIOMETRIA MÉDIA INICIAL DOS ALEVINOS NOS DOIS ANOS EXPERIMENTAIS}

\begin{tabular}{lccrc}
\hline & \multicolumn{1}{c}{ Ano 1 } & & Ano 2 \\
\hline ALEVINOS & Peso $(\mathrm{g})$ & Comprimento $(\mathrm{cm})$ & \multicolumn{1}{c}{ Peso $(\mathrm{g})$} & Comprimento $(\mathrm{cm})$ \\
\hline Húngara & $10,60 \pm 0,82$ & $9,10 \pm 0,20$ & $6,80 \pm 1,27$ & $7,70 \pm 0,40$ \\
Capim & $10,21 \pm 2,72$ & $10,28 \pm 0,49$ & $16,20 \pm 4,10$ & $11,90 \pm 1,07$ \\
Cabeça grande & $19,75 \pm 0,25$ & $12,20 \pm 0,47$ & $12,30 \pm 2,56$ & $10,30 \pm 0,80$ \\
\hline
\end{tabular}

Durante a realização do experimento foram coletadas amostras de água e enviadas para o Laboratório de Análises de Resíduos de Pesticidas (LARP) da UFSM. No ano 1, as coletas ocorreram antes da aplicação dos agroquímicos e entre os dias $1^{\circ}$ a $36^{\circ}$ e no ano 2 do $1^{\circ}$ ao $56^{\circ}$ dia, a cada dois dias, sendo as amostras analisadas segundo ZANELLA et al. (2000). Os parâmetros físico-químicos da água foram monitorados semanalmente na área do refúgio: $\mathrm{pH}$ (pHmetro), dureza total (APHA, 1992), temperatura e oxigênio dissolvido (Oxímetro Oaktron) e transparência da água (Disco de Secchi).

Realizou-se a avaliação da infestação e do controle de bicheira-da-raiz nos tratamentos ( $T 1, T 2$, T3, T6 e T8) mediante coleta de três amostras de solo e raízes por parcela, 30 dias após a aplicação dos tratamentos. Em cada parcela foram retiradas três amostras de solo e raízes, usando-se secção de cano de PVC com $10 \mathrm{~cm}$ de diâmetro e $20 \mathrm{~cm}$ de altura até $8 \mathrm{~cm}$ no solo. As amostras foram agitadas sob água em peneira apropriada para liberação e contagem das larvas e pupas (TUGWELLe STEPHEN, 1981). A contagem da população inicial de plantas de arroz foi realizada aos 16 dias após a semeadura, juntamente com a avaliação de plantas daninhas. Por ocasião da colheita foram avaliadas aleatoriamente 10 plantas para determinar sua estatura e coletadas as panículas para estimar o número de grãos por panícula, a esterilidade de espiguetas e a massa de mil grãos. A produtividade de grãos do arroz foi obtida pela extrapolação da massa de grãos, provenientes da colheita em área de $6 \mathrm{~m}^{-2}$. Ressalta-se que a construção de refúgios no sistema de rizipiscultura promove redução da área útil de produção de arroz, que na situação do presente estudo correspondeu a $8 \%$ nos tratamentos com peixes. Após a colheita do arroz manteve-se a altura de lâmina de água de $20-25 \mathrm{~cm}$ nas parcelas até a próxima semeadura, ocasião em que se procedeu a drenagem dos refúgios e a retirada dos peixes para biometria. Os dados expressos em percentagem foram transformados para arcoseno e todos os resultados submetidos à análise de variância, sendo as médias comparadas pelo teste de Tukey ao nível de $5 \%$ de probabilidade de erro (BANZATO e KRONCA, 1995).

\section{RESULTADOS E DISCUSSÃO}

A produtividade média do arroz irrigado no ano 1 foi de $4151 \mathrm{~kg} \mathrm{ha}^{-1}$ (Tabela 2). Verificou-se influência dos tratamentos utilizados, especialmente pela competição com as plantas daninhas, 
apesar dos valores serem estatisticamente semelhantes à aplicação isolada de cada produto. Os tratamentos T3, T7 e T8 que não receberam herbicida obtiveram as menores produtividades de arroz. No sistema pré-germinado de cultivo de arroz, com lâmina contínua de água, é fundamental que se tenha o controle da altura de lâmina como forma de emergência das plantas daninhas (especialmente gramíneas). O levantamento da população inicial de plantas daninhas nesse ano demonstrou a presença de 371 plantas $\mathrm{m}^{-2}$, evidenciando a necessidade de controle que pode ser observada pelos resultados de produtividade obtidos. As espécies daninhas encontradas foram: 39\% de Sagittaria sp. (sagitária), 23\% de Heteranthera sp. (agrião-do-brejo), 16\% de Cyperus sp. (junquinho), 12\% de Ludwigia sp. (cruz-de-malta) e 10\% de Fimbristylis sp. (cuminho).

\section{TABELA 2 - PRODUTIVIDADE, ESTANDE INICIAL, ESTATURA DE PLANTAS, GRÃOS POR PANÍCULA, MASSA DE MIL GRÃOS (MMG) E ESTERILIDADE DE ESPIGUETAS DA CULTURA DO ARROZ IRRIGADO EM FUNÇÃO DOS TRATAMENTOS PROPOSTOS EM ÁREA DE RIZIPISCICULTURA}

\begin{tabular}{lcccccc}
\hline Tratamento & $\begin{array}{c}\text { Produt. } \\
\left(\mathrm{kg} \mathrm{ha}^{-1}\right)\end{array}$ & $\begin{array}{c}\text { Estande } \\
\left(\mathrm{pl} \mathrm{m}^{-2}\right)\end{array}$ & $\begin{array}{c}\text { Estatura } \\
(\mathrm{cm})\end{array}$ & $\begin{array}{c}\text { Grãos/Pan. } \\
2003 / 2004\end{array}$ & $\begin{array}{c}\text { MMG } \\
(\mathrm{g})\end{array}$ & $\begin{array}{c}\text { Esteril. }^{1} \\
(\%)\end{array}$ \\
\hline T1 (A+P) & $4240 \mathrm{ab}^{*}$ & $252^{\mathrm{ns}}$ & $65 \mathrm{ab}$ & $74,2 \mathrm{ab}$ & $25,8^{\mathrm{ns}}$ & $12,8^{\mathrm{ns}}$ \\
T2 (M+P) & $4543 \mathrm{a}$ & 320 & $65 \mathrm{ab}$ & $71,0 \mathrm{ab}$ & 25,7 & 11,7 \\
T3 (C+P) & $3602 \mathrm{abc}$ & 272 & $65 \mathrm{ab}$ & $73,6 \mathrm{ab}$ & 26,1 & 14,5 \\
T4 (A+M+C+P) & $5424 \mathrm{a}$ & 300 & $68 \mathrm{ab}$ & $77,4 \mathrm{a}$ & 26,2 & 23,9 \\
T5 (A+M+C) & $+5515 \mathrm{a}$ & 240 & $69 \mathrm{a}$ & $75,1 \mathrm{ab}$ & 26,2 & 13,3 \\
T6 (A+M+P) & $5663 \mathrm{a}$ & 268 & $68 \mathrm{ab}$ & $77,3 \mathrm{a}$ & 25,9 & 11,9 \\
T7 (Ar) & $+2114 \mathrm{bc}$ & 308 & $59 \mathrm{~b}$ & $58,3 \mathrm{ab}$ & 24,8 & 15,1 \\
T8 (Ar+P) & $2104 \mathrm{c}$ & 247 & $58 \mathrm{~b}$ & $52,6 \mathrm{~b}$ & 25,0 & 12,7 \\
\hline Média & 4151 & 276 & 65 & 69,9 & 25,7 & 14,5 \\
CV (\%) & 21,6 & 15,1 & 6,6 & 14,0 & 3,3 & 17,1 \\
\hline & & & & $2004 / 2005$ & & \\
\hline T1 (A+P) & $5121 \mathrm{~ns}$ & $2655^{\mathrm{ns}}$ & $66^{\mathrm{ns}}$ & $74,2 \mathrm{~ns}$ & 24,9 ns & $18,6{ }^{\mathrm{ns}}$ \\
T2 (M+P) & 5347 & 235 & 67 & 71,0 & 25,0 & 14,9 \\
T3 (C+P) & 6101 & 255 & 67 & 73,6 & 25,8 & 16,8 \\
T4 (A+M+C+P) & 6167 & 340 & 65 & 77,4 & 25,4 & 20,0 \\
T5 (A+M+C) & +5961 & 290 & 67 & 75,1 & 25,7 & 17,9 \\
T6 (A+M+P) & 5620 & 370 & 65 & 77,3 & 25,4 & 15,6 \\
T7 (Ar) & +5194 & 300 & 64 & 58,3 & 24,9 & 16,7 \\
T8 (Ar+P) & 5632 & 325 & 67 & 52,6 & 25,3 & 17,1 \\
\hline Média & 5643 & 297 & 66 & 82,6 & 25,3 & 17,2 \\
CV (\%) & 15,9 & 38,6 & 4,1 & 13,0 & 1,7 & 14,3 \\
\hline
\end{tabular}

nsTratamentos não diferem pelo Teste F.

*Médias seguidas por letras distintas diferem entre si pelo teste de Tukey ao nível de 5\% de probabilidade de erro.

(A) Azinsulfurom; (M) Metsulfurom-metílico; (C) Carbofurano; (P) Rizipiscicultura; (Ar) Arroz. +acréscimo de $8 \%$ no rendimento do arroz devido a área do refúgio. ${ }^{1}$ Esterilidade (dados transformados por arcoseno). CV = Coeficiente de Variação.

Comparando os resultados do T6 (herbicidas e peixe) com o T4 (todos os agroquímicos e peixe) no ano 1 não houve efeito adicional nos níveis produtivos obtidos quando se aplicou 0 inseticida. Entretanto, na média dos tratamentos sem utilização de carbofurano quantificou-se no levantamento da infestação da bicheira-da-raiz 7 larvas e 2 pupas por amostragem. As recomendações técnicas da cultura do arroz para o Sul do Brasil (SOSBAI, 2005) especificam que acima de certo nível populacional (cerca de 5 larvas), e dependendo da cultivar, a cada larva a mais por amostra é esperada redução de 1,1 a 1,5\% na produção de grãos de cultivares de ciclo médio e precoce, respectivamente.

O estande de plantas de arroz foi de 276 plantas $\mathrm{m}^{-2}$ (Tabela 2), não havendo diferença significativa entre os tratamentos. A estatura média de plantas no ano 1 foi de $65 \mathrm{~cm}$, sendo que nos 
tratamentos em que não houve utilização de nenhum agroquímico obteve-se menor estatura. Nesses tratamentos verificou-se menor número de grãos por panícula, o que está coerente com a produtividade advinda dos mesmos.

A produtividade média do arroz irrigado no ano 2 foi de $5643 \mathrm{~kg} \mathrm{ha}^{-1}$ (Tabela 2), não havendo diferença significativa entre os tratamentos e entre os demais parâmetros avaliados da cultura. $\mathrm{O}$ levantamento da população de plantas daninhas demonstrou a presença de 41 plantas $\mathrm{m}^{-2}$, sendo $82 \%$ de Sagittaria sp. (sagitária), 9\% de Heteranthera sp. (agrião-do-brejo), 7\% de Echinochloa sp. (capimarroz) e $2 \%$ de Ludwigia sp. (cruz-de-malta). Essa redução pode ser devida a aplicações de herbicida de ação total em todos os tratamentos antes da semeadura do arroz no ano 2, além da lâmina de água que se manteve em nível adequado em todas as parcelas. No levantamento da bicheira-da-raiz foram encontradas 2 larvas e 4 pupas por amostragem na média dos tratamentos sem carbofurano.

Os resultados de produção de arroz em área de rizipiscicultura são bastante variáveis. ROTHUIS et al. (1998 a) realizaram experimentos na China em rizipiscicultura, usando carpas (Puntius gonionotus e C. carpio) e tilápia do Nilo (Oreochromis niloticus). Encontraram produtividade média de arroz de $2817 \mathrm{~kg} \mathrm{ha}^{-1} \mathrm{e}$ não observaram diferença entre os tratamentos com e sem peixes. Em outro trabalho, com as mesmas espécies de peixes, ROTHUIS et al. (1999) obtiveram maior produtividade média do arroz ( $5550 \mathrm{~kg} \mathrm{ha}^{-1}$ ). SATO (2002), utilizando carpas (C. idella e C. carpio) e tilápia do Nilo (O. niloticus) em Santa Catarina (Brasil), encontrou produtividade média do arroz nos locais com peixes de $5166 \mathrm{~kg} \mathrm{ha}^{-1} \mathrm{e}$ no tratamento testemunha de $4201 \mathrm{~kg} \mathrm{ha}^{-1}$. MARCHEZAN et al. (2006) trabalharam com policultivo de carpas (C. carpio, C. idella, A. nobilis e H. molitrix) e jundiá (Rhamdia quelen) em rizipiscicultura no Rio Grande do Sul (Brasil). Relataram produtividade de $6372 \mathrm{~kg} \mathrm{ha}^{-1}$ de arroz, utilizando adequada manutenção da lâmina d'água para a supressão do desenvolvimento de plantas daninhas de folhas estreitas e os herbicidas azinsulfurom e metsulfurommetílico para o controle de plantas aquáticas e ciperáceas.

Com relação à presença de peixes na área de arroz, os resultados demonstram baixas taxas de sobrevivência das carpas ao final de cada ano experimental. No ano 1 foi constatado 1,43\% de sobrevivência dos peixes do total colocado. Os valores médios ( $\mathrm{T} 1=1,75 ; \mathrm{T} 2=1,25 ; \mathrm{T} 3=1 ; \mathrm{T} 4=0,5$; $\mathrm{T} 6=1,25$ e T8 $=2,5$ peixes por tratamento) são representados pelas carpas capim e cabeça grande, não sendo despescada nenhuma carpa húngara. No ano 2 , a percentagem foi ainda menor com apenas $0,30 \%$ de sobrevivência $(\mathrm{T} 1=0,43 ; \mathrm{T} 2=0,43 ; \mathrm{T} 3=0,0 ; \mathrm{T} 4=0,0 ; \mathrm{T} 6=0,0$ e T8 $=0,87$ peixe(s) por tratamento), representada apenas pela carpa capim. Entretanto, SATO (2002) verificou sobrevivência média dos peixes em rizipiscicultura de 65\%, MARCHEZAN et al. (2006) de $41 \%$ e ROTHUIS et al. (1999) de 79\%. Acredita-se que a baixa sobrevivência das espécies tenha sido fortemente influenciada pela ocorrência de predadores na área experimental como, aves (garças - Casmerodius sp.), répteis (jacarés - Caiman sp.) e mamíferos (lontras - Lutra sp.- principal predador noturno de peixes). Verificouse que os agroquímicos não afetaram a sobrevivência dos peixes, pois a mortalidade pela ação desses foi nula nos tanques-rede colocados em cada refúgio das parcelas. Entretanto, foi observada mortalidade das espécies de carpas nessas estruturas devido a predação por aves (garças).

Na primeira coleta de água, realizada aproximadamente 12 horas após a aplicação de azinsulfurom e metsulfurom-metílico, não foi quantificada a presença desses herbicidas nos dois anos (limite de detecção - LOD $=<0,001 \mathrm{mg} \mathrm{L}^{-1}$ ). Experimentos realizados concomitantemente em laboratório (GOLOMBIESKI et al., 2005, dados não-publicados) demonstraram que esses herbicidas não afetam a sobrevivência das carpas. Mesmo em testes de concentração letal mediana $\left(\mathrm{CL}_{50} ; 96 \mathrm{~h}\right)$, doses de até $200 \mathrm{mg} \mathrm{L}^{-1}$ não provocaram mortalidade nem alteraram a alimentação e a natação das carpas. Nesse caso, os índices de segurança são de 40.000 para azinsulfurom e 100.000 para metsulfurom-metílico, superiores aos aplicados no experimento em campo (0,005 e 0,002 $\mathrm{mg} \mathrm{L}^{-1}$, respectivamente). Já o carbofurano foi detectado na lavoura de arroz aos 16 dias após sua aplicação no ano $1\left(0,022 \mathrm{mg} \mathrm{L}^{-1}\right)$ e 17 dias no ano $2\left(0,013 \mathrm{mg} \mathrm{L}^{-1}\right)$. GOLOMBIESKI et al. (2006) em testes de concentração letal mediana $\left(\mathrm{CL}_{50} ; 96 \mathrm{~h}\right)$, realizados em laboratório, encontraram: $1,81 \mathrm{mg} \mathrm{L}^{-1}$ do inseticida carbofurano para carpa húngara; 2,61 $\mathrm{mg} \mathrm{L}^{-1}$ para carpa capim e 2,37 $\mathrm{mg} \mathrm{L}^{-1}$ para carpa cabeça grande com índices de segurança de 2,$41 ; 6,31$ e 3,16, respectivamente. Segundo SOLOMON (1997), índices de segurança inferiores 
a 20 demonstram produtos com maior risco de impacto ambiental. Tal fato foi confirmado mediante alterações alimentares e natatórias das carpas, bem como mudanças na atividade enzimática e letargia em baixas concentrações testadas $\left(0,5 \mathrm{mg} \mathrm{L}^{-1}\right.$ para carpas húngara e cabeça grande). Assim, esse inseticida apresenta maior potencial de risco e sua utilização na lavoura com rizipiscicultura exige cuidado. No presente trabalho, as carpas foram colocadas nas parcelas sete dias após a aplicação dos tratamentos. As concentrações de carbofurano na água foram de 0,025 e 0,0144 $\mathrm{mg} \mathrm{L}^{-1}$ nos anos experimentais 1 e 2, respectivamente, níveis que podem ser considerados pouco tóxicos para essas espécies de peixe.

Os resultados dos parâmetros físico-químicos da água (Tabela 3) demonstram que para o ano 1 houve diferença significativa entre os tratamentos nos níveis de oxigênio dissolvido, $\mathrm{pH}$ e transparência da água no primeiro período avaliado. A concentração de oxigênio dissolvido na testemunha 1 (T7) apresentou-se mais elevada devido ao menor consumo desse gás, já que não havia a presença de peixes. Contudo, tais valores mantiveram-se baixos nesse período, possivelmente associados com as altas temperaturas da água. A transparência da água foi maior nos tratamentos sem peixes (T5 e T7). A presença principalmente da espécie carpa húngara, que remove o solo à procura de alimentos, deixa a água turva com maior quantidade de materiais sólidos em suspensão. No segundo período do ano 1 não houve diferença significativa nos parâmetros avaliados entre os tratamentos até o final do experimento, possivelmente pela baixa densidade de peixes na área em razão da predação.

\section{TABELA 3 - PARÂMETROS FÍSICO-QUÍMICOS DA ÁGUA MONITORADOS DURANTE O EXPERIMENTO (2003/2004) EM FUNÇÃO DOS TRATAMENTOS PROPOSTOS EM ÁREA DE RIZIPISCICULTURA}

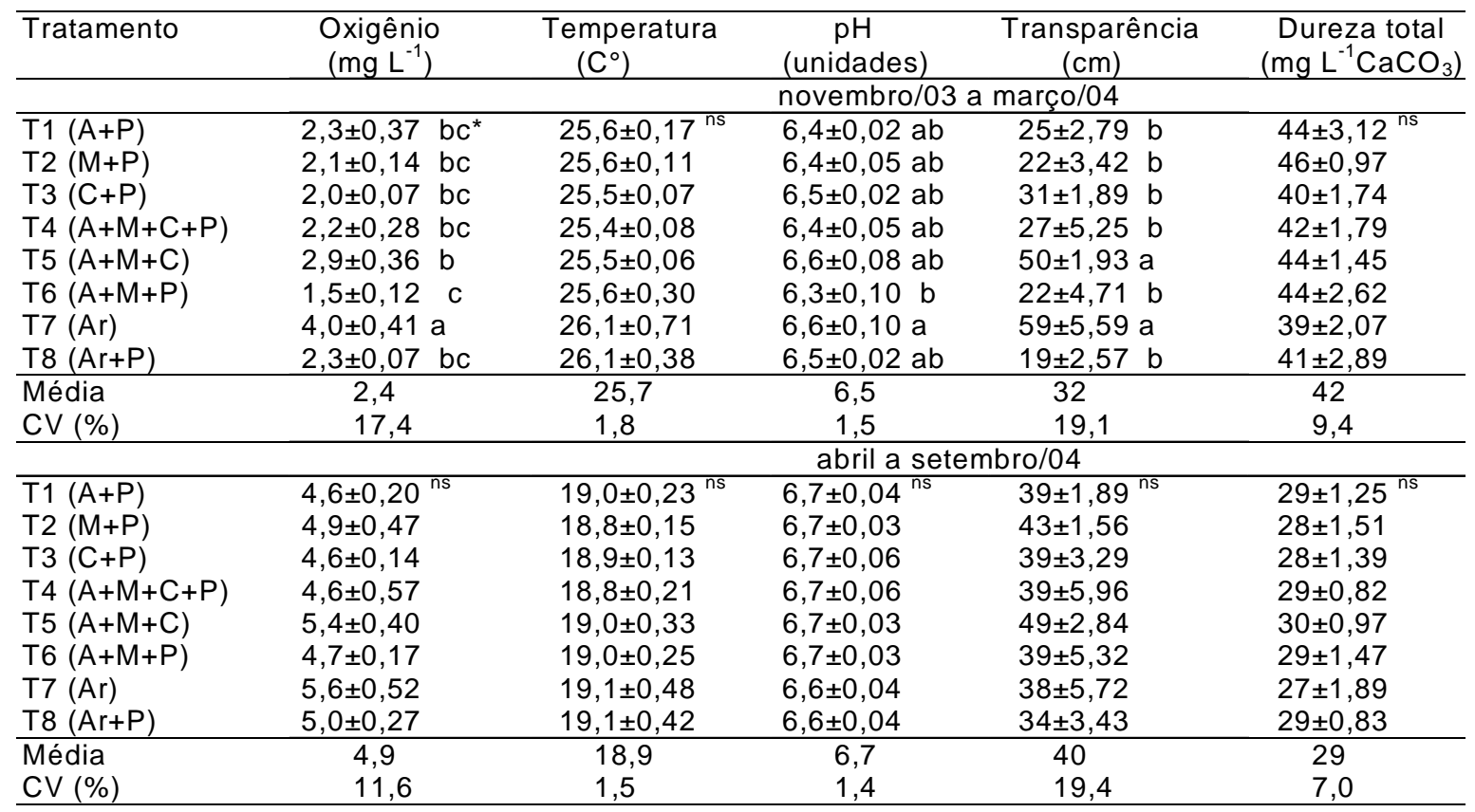

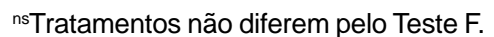

*Médias seguidas por letras distintas diferem entre si pelo teste de Tukey em nível de $5 \%$ de probabilidade de erro. (A) Azinsulfurom; (M) Metsulfurom-metílico; (C) Carbofurano; (P) Rizipiscicultura; (Ar)Arroz. CV = Coeficiente de Variação.

No ano 2 houve diferença significativa para os níveis de oxigênio dissolvido e transparência da água no primeiro período (dezembro a março), sendo esses valores mais altos no tratamento T5 e também T7 para o oxigênio (Tabela 4). Os níveis de oxigênio dissolvido foram maiores nesse período nos tratamentos com ausência de peixes. Entretanto, no segundo período não foi verificada diferença significativa entre os tratamentos. 


\section{TABELA 4 - PARÂMETROS FÍSICO-QUÍMICOS DA ÁGUA MONITORADOS ENTRE DEZEMBRO DE 2004 E JULHO DE 2005 EM FUNÇÃO DOS TRATAMENTOS EM ÁREA DE RIZIPISCICULTURA}

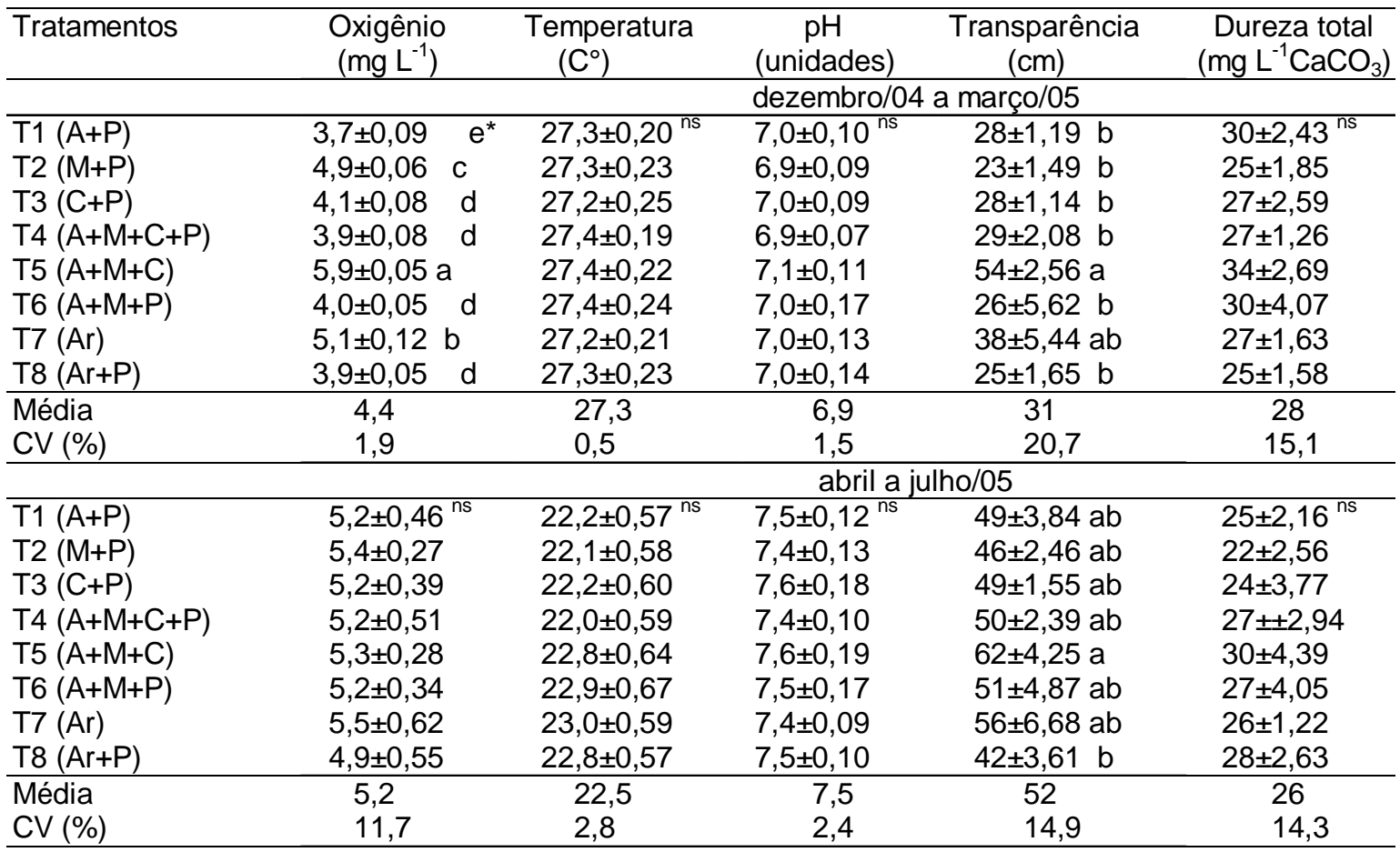

${ }^{n s}$ Tratamentos não diferem pelo Teste F.

*Médias seguidas por letras distintas diferem entre si pelo teste de Tukey em nível de 5\% de probabilidade de erro. (A)Azinsulfurom; (M) Metsulfurom-metílico; (C) Carbofurano; (P) Rizipiscicultura; (Ar) arroz. CV = Coeficiente de variação.

GOLOMBIESKI et al. (2005) demonstraram que as características físico-químicas da água não afetam o crescimento e o desenvolvimento dos peixes no consórcio com arroz irrigado para as espécies cultivadas (60\% de carpa húngara, $20 \%$ de carpa capim, $10 \%$ de jundiá, $5 \%$ de carpa prateada e $5 \%$ de carpa cabeça grande).

Neste experimento, os parâmetros físico-químicos enquadram-se nos limites aceitáveis para a criação de peixes em rizipiscicultura.

\section{CONCLUSÃO}

A aplicação dos agroquímicos (carbofurano, azinsulfurom e metsulfurom-metílico) na lavoura arrozeira não afetou a sobrevivência de carpas húngara, capim e cabeça grande quando os alevinos foram introduzidos sete dias após sua aplicação. A produtividade do arroz não foi influenciada pelos agroquímicos, quando se utilizou manejo adequado de lâmina contínua a partir da semeadura prégerminada do arroz.

\section{ABSTRACT \\ EFFECTS OF CARBOFURAN, METSULFURON-METHYL AND AZIMSULFURON ON CARPS SURVIVAL AND RICE AND FISH PRODUCTION IN RICE-FISH CULTURE}

This study was carried out to evaluate the fingerlings carps survival exposure to carbofuran, metsulfuron-methyl and azimsulfuron in rice-fish culture, as well as irrigate rice and fish production. Common, grass and bighead fingerlings carps were exposed to carbofuran, metsulfuron-methyl and azimsulfuron agrochemicals in pre-germinated rice production system with continual flooding water management during 2003/04 and 2004/05 growing season. 
Randomized complete block design with eight treatments and four repetitions, associating carps under the presence and absent of agrochemicals was used. Seven days after agrochemicals application, fingerlings carps were put in the area and it was verified that agrochemicals did not affect these survival. Due to use of continual flooding water management system, the rice yield grain is not affected by agrochemicals utilization.

KEY-WORDS: Cyprinus carpio; Ctenopharyngodon idella; Aristichthys nobilis; RICE-FISH CULTURE; CARBOFURAN; METSULFURON-METHYL; AZIMSULFURON.

\section{REFERÊNCIAS}

1 APHA. American Public Health Association. Standard methods for examination of water and wastewater. $18^{\text {th }}$ ed. Virgínia, 1992. 1155 p.

2 BANZATO, D. A.; KRONCA, S. N. Experimentação agrícola. 3. ed. Jaboticabal: FUNEP, 1995. 247 p.

3 COTRIM, D.S. et al. Rizipiscicultura: um sistema agroecológico de produção. In: CONGRESSO DA CADEIA PRODUTIVA DO ARROZ. REUNIÃO NACIONAL DE PESQUISA DEARROZ, 7., Florianópolis, 2002. Anais... Florianópolis: 2002. p. 690-693.

4 GOLOMBIESKI, J.I. et al. Qualidade da água no consórcio de peixes com arroz irrigado. Ciência Rural, v. 6, n. 35, p. 1263-1268, 2005.

5 GOLOMBIESKI, J.I. et al. Acetylcholinesterase activity in the brain and muscle of Cyprinus carpio, Ctenopharyngodon idella, and Aristichthys nobilis after acute exposure to carbofuran. Scientia Agricola, 2007 (submetido).

6 MARTINS, J.F.S. et al. Descrição e manejo integrado de insetos-praga em arroz irigado. In: GOMES, A.S.; JÚNIOR, A.M.M. Arroz Irrigado no Sul do Brasil. Brasília, DF: Embrapa Informação Tecnológica. 2004. Cap. 19. p. 635-675.

7 MARCHEZAN, E. et al. Produção integrada de arroz e peixes. Ciência Rural, v. 36, n. 2, p. 411-417, 2006.

8 ROTHUIS, A.J. et al. Polyculture of silver barb, Puntius gonionotus (Bleeker), Nile tilapia, Oreochromis niloticus (L.), and common carp, Cyprinus carpio L., in Vietnamese ricefields: feeding ecology and impact on rice and ricefields environment. Aquaculture Research, v. 29, p. 649-660, 1998.

9 ROTHUIS, A.J. et al. The effect of rice seeding rate on rice and fish production, and weed abundance in direct-seeded rice-fish culture. Aquaculture, v. 172, p. 255-274, 1999.

10 SATO, G. Rizipiscicultura: uma alternativa rentável para o produtor de arroz irrigado. Agropecuária Catarinense, v. 15, n. 3, p. 47-50, 2002.

11 SOLOMON, K.R. Advances in the evaluation of the toxicological risks of herbicides to the environment. In: CONGRESSO BRASILEIRO DA CIÊNCIA DAS PLANTAS DANINHAS, 21., Caxambu, MG. Anais... Caxambu: SBCPD, 1997. p. 163-172.

12 SOSBAI. Sociedade Sul-Brasileira de Arroz Irrigado. Arroz Irrigado: recomendações técnicas da pesquisa para ao sul do Brasil. Balneário Camboriú, SC, 2003. 126 p.

13 SOSBAI. Sociedade Sul-Brasileira de Arroz Irrigado. Arroz Irrigado: recomendações técnicas da pesquisa para ao sul do Brasil. Santa Maria, 2005. 159 p.

14 TUGWELL, N.P.; STEPHEN, F.M. Rice water weevil seasonal abundance, economic levels, and sequencial sampling plans. Fayeltville: Agricultural Experiment Station, 1981. 16 p. (Bulletin, 849).

15 ZANELLA, R. et al. Development and validation of a high-performace liquid chromatographic procedure for the determination of clomazone residues in surface water. J. Chromatogr. A., v. 904, p. 257-262, 2000.

\section{AGRADECIMENTOS}

Os autores agradecem ao Conselho Nacional de Desenvolvimento Científico e Tecnológico pelo financiamento parcial deste trabalho e ao Grupo de Pesquisa em Arroz e Uso Alternativo de Várzea da UFSM pela assistência durante a realização dos experimentos. 